\title{
Forward Speed Control of a Pulsed-Jet Soft-Bodied Underwater Vehicle
}

\author{
Giorelli Michele, Student Member, IEEE, Giorgio-Serchi Francesco and Laschi Cecilia, Senior Member, IEEE
}

\begin{abstract}
This paper reports on the development of the control for a new class of soft underwater vehicles. These vehicles exploit their soft-bodied nature to produce thrust by cyclically ingesting and expelling ambient fluid. A forward speed control based on the linearised dynamics of the robot is design. The control succeeds at dealing with the discontinuous thrust by accounting for the shape-change driven actuation.
\end{abstract}

\section{INTRODUCTION}

Sectors associated with marine operations are in perpetual growth. Offshore construction, deep ocean drilling, maritime and, lately, renewable energies harvesting technologies from ocean wind and wave farms bring evidence that seas remain an immense resource for economic growth. As a consequence, technologies which provide aid during standard operations at sea or even enable activities which are, at present, infeasible, should be regarded as greatly beneficial.

Among the major constraints for offshore and underwater operations lies the difficulty of performing tasks, such as survey or construction, in close proximity to floating or submerged structures during hazardous conditions, as it occurs in wave or current dominated environments. In response to the need for enhanced capability to deal with complex scenarios, a growing effort in the scientific and engineering community has been dedicated to the development of vehicles with augmented manoeuvrability [1]. The concept of fast response, highly manoeuvrable marine vehicles has largely benefited from the study of marine animals which sport outstanding manoeuvring skills such as fast-start manoeuvres in fish, [2] and escapejetting in cephalopods, [3]. This has fostered a significant effort in studying bioinspired modes of locomotion based on finned or undulatory, snake-like propulsion. In recent times, the study of pulsed-jet propulsion has gained popularity in the scientific community for the degree of manouverability which it enables [4], [6] and [5]. In [7] pulsed-jet swimming has been incorporated in a soft-bodied vehicle which not only takes advantages of the assets of vortex-enhanced propulsion [9], but it also benefits from a highly compliant structural design, which makes it less prone to causing or suffering from damage due to impact, thus making it highly suited for operations in cramped environments and highly perturbed environmental conditions. This work deals with the first attempt to control

This work was jointly supported by the EU Commission in the frame of the CFD-OctoProp Project FP7 European Reintegration Grant and the Foundation Grant project PoseiDRONE supported by the Cassa di Risparmi di Livorno. All the authors are with Centre for Sea Technologies and Marine Robotics, The BioRobotics Institute, Scuola Superiore Sant'Anna, Livorno, Italy, 57126, e-mail: m.giorelli@sssup.it, f.serchi@sssup.it, cecilia.laschi@sssup.it

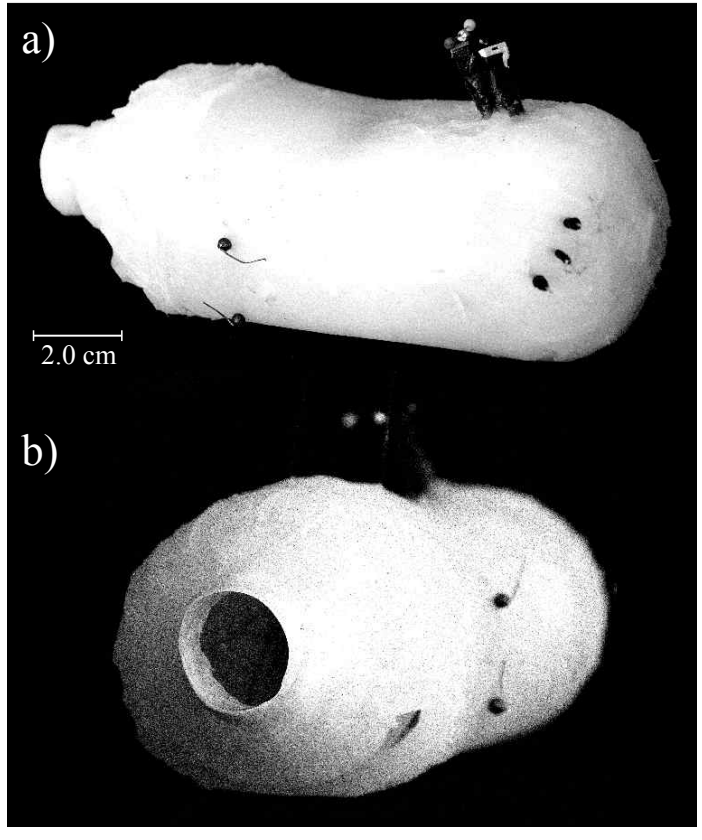

Fig. 1. Rear $(a)$ and frontal $(b)$ view of the Soft Unmanned Underwater Vehicle.

this kind of vehicle by accounting for its unconventional design and thrust production routine.

\section{The Soft Unmanned Underwater Vehicle}

The vehicle, Fig. 1, is composed of a hollow shell of rubberlike material. A schematic portray of its internal constituents is provided in Fig. 2. Two distinct apertures are dedicated respectively to the ingestion, i.e. ingestion valve (element 9 in Fig. 2.a), and the expulsion (element 6 in Fig. 2.a), i.e. the nozzle or siphon, of ambient water. The collapse of the shell is actuated via cable transmission: a set of cables (elements 4 in Fig. 2) are attached, at one end, to the external wall of the silicon shell (element 5 in Fig. 2) and, at the other end, to a crank (element 8 in Fig. 2) fitted onto the shaft of a gearmotor (element 7 in Fig. 2) hosted inside the shell. The rotation of the shaft cyclically pulls and releases the cables which, in turn, pull and release the elastic walls of the shell. When the elastic walls are pulled inward, the water stored in the shell gets pressurized and issues abruptly across the nozzle accelerating the vehicle in the opposite direction. Then the cables are released and the stresses generated within the 
a) 1

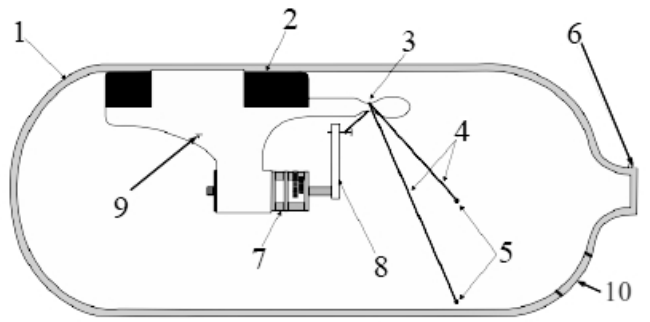

b)

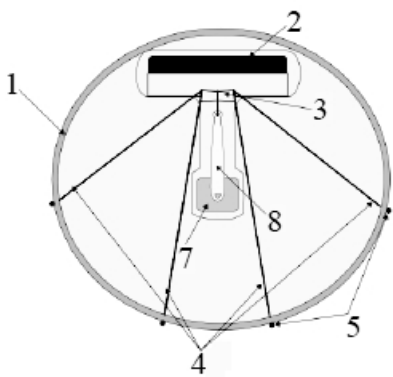

Fig. 2. Schematic depiction of the lateral (a) and frontal (b) view of the vehicle. The numbers refer to: (1) the rubber-like shell, (2) the batteries, (3) the fixed pulley, or fairlead, (4) the cables, (5) cable attachments, (6) outflow nozzle, (7) the gearmotor, (8) the crank, (9) supportive structure, (10) inflow orifice.

elastic walls bring the shell back to the unstrained state. The pressure drop generated in this way inside the cavity sucks ambient fluid across the ingestion valve, refilling the shell. This sequence determines a single pulsation of the vehicle. This specific vehicle has been tested in water and preliminary results from an early series of experiments is reported in [8].

\section{Dynamics AND Thrust Modelling}

In [8] the dynamics of the soft underwater vehicle is devised with a focus on the thrust production routine. The model enables the estimation of the dynamics of the vehicle according to the thrust produced via the cable-induced deformation of the elastic shell. The thrust is defined as $\tau=q \dot{m}$ with $q$ being the outflow across the nozzle-exit area $A_{n}$ and $\dot{m}$ being the variation of mass occurring within the shell due to the cabledriven collapse of the elastic chamber. This is expressed by,

$$
\begin{aligned}
\dot{m}(t) & =\rho \frac{d\left(\Delta_{s} A_{s}\right)}{d t}=\frac{d \Delta_{s}}{d t} A_{s}+\Delta_{s} \frac{d A_{s}}{d t} \\
\frac{d \Delta_{s}}{d t} & =2 l \omega \cos \frac{\alpha}{2} \\
\frac{d A_{s}}{d t} & =\frac{d A_{s}}{d \alpha} \frac{d \alpha}{d t}=\frac{d A_{s}}{d \alpha} \omega
\end{aligned}
$$

where $A_{s}$ and $\Delta_{s}$ respectively define the time dependent cross sectional area being sqeezed by the cable-driven collapse and axial extent of the shell affected by the cable-induced strain, while $l, \omega$ and $\alpha$ are the length of the crank, the angular velocity and position of the motor. The $q$ term is defined relative to the ambient fluid velocity $u$, yielding,

$$
q=\frac{1}{\rho A_{n}} \dot{m}-u \text {. }
$$

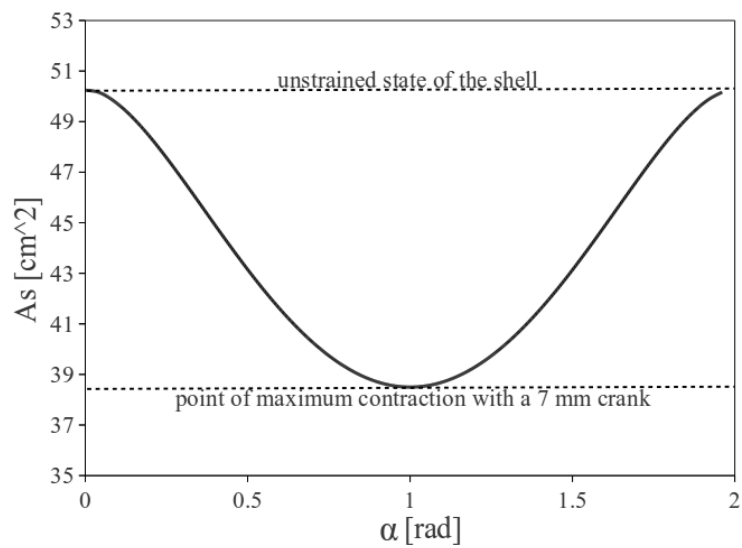

Fig. 3. Profile of the cross sectional area $A_{s}$ of a $4 \mathrm{~cm}$ radius cylindrical shell as a function of the angular position $\alpha$ of a $7 \mathrm{~mm}$ long crank with 4 cables attached to.

Therefore, by substituting (1) into (4), an expression for the thrust is derived,

$$
\tau=\left[\frac{1}{A_{n}} \frac{d}{d t}\left(\Delta_{s} A_{s}\right)-u\right] \rho \frac{d}{d t}\left(\Delta_{s} A_{s}\right) .
$$

Closure of this equation requires estimation of $A_{s} . A_{s}$ is estimated through a kinematic representation of the cross sectional deformation of a rubber-like cylindrical shell subject to pointwise inward radial loading. The interested reader is referred to [8] for further details on this specific aspect. For the reminder of this work, however, $A_{s}$ is provided as a prescribed profile computed with the model described in [8], see Fig. 3. This profile corresponds to a $4 \mathrm{~cm}$ radius cylindrical shell with 4 equispaced cables attached along a single cross section of the shell and driven by a $7 \mathrm{~mm}$ crank. As dictated by design specification, contraction of the cables occurs for $0<\alpha<\pi$. During this phase, the shell gets collapsed and the cross sectional area $A_{s}$ shrinks. For $\pi<\alpha<2 \pi$ the cables are released and the shell inflates back to its original unstrained state, bringing $A_{s}$ to its initial value.

In order to estimate the load torque which the motor is subject to while collapsing the silicone shell, a simple experiment is devised. A mock-up of the vehicle made of a
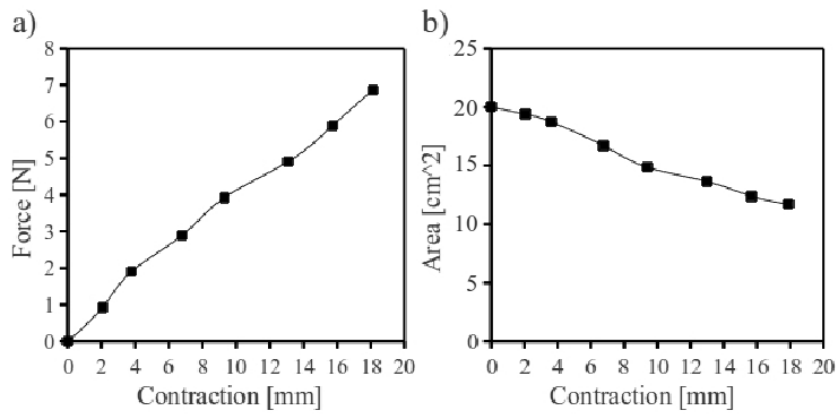

Fig. 4. Results from experimental analysis on the contraction of a $5 \mathrm{~mm}$ thick silicone shell radially collapsed by 4 cables. In $(a)$, the force required to shorten 4 cables is plotted, in $(b)$ the change in cross sectional area due to the cable contraction is reported. 
$2.5 \mathrm{~cm}$ radius and $5 \mathrm{~mm}$ thick cylindrical shell is moulded. The shell hosts 4 cables attached at equispaced spots along the external wall of the shell and gathered at the center of the cylinder. This set up enables an accurate measuring of the cross sectional reduction due to cable shortening and tension on the cables. The results from experiments performed in air are presented in Fig.4.a-b and will be discussed later in section $\mathrm{V}$.

\section{CONTROL}

In a recent work [10], a position controller fed by sinusoidal reference signals of an underwater robot with a vortex ring thrust was presented. Here, a forward speed controller is designed for this new soft bodied vehicle. In order to derive a suitable control, equation (5) is manipulated until the following is achieved:

$$
\begin{aligned}
\tau & =X_{\omega^{2}}(\alpha) \omega^{2}+X_{u \omega}(\alpha) u \omega \\
X_{\omega^{2}}(\alpha) & =\rho 4 l^{2} g(\alpha)^{2} / A_{n} \\
X_{u \omega}(\alpha) & =-\rho 2 \lg (\alpha) \\
g(\alpha) & =\cos \frac{\alpha}{2} A_{s}+\frac{d A_{s}}{d \alpha} \sin \frac{\alpha}{2}
\end{aligned}
$$

Therefore, the one-dimensional momentum equation for a neutrally buoyant, rigid body translating in water and propelled by the actuation principle described above reads as follows:

$$
\tilde{M} \dot{u}=-X_{u^{2}} u^{2}+X_{\omega^{2}}(\alpha) \omega^{2}+X_{u \omega}(\alpha) u \omega
$$

where $\tilde{M}$ includes the mass of the vehicle and the axial added mass coefficient, and $X_{u^{2}}$ is the quadratic drag term of a bullet-like shaped body with a mildly rough external surface.

In order to design a controller some simplifications have been studied and tested. The time-variant terms $X_{w^{2}}(\alpha)$ and $X_{u w}(\alpha)$ are substituted by the mean values $\bar{X}_{w^{2}}$ and $\bar{X}_{u w}$. A second simplification consists in the linearisation of the dynamic model at the nominal motor speed $\bar{\omega}$ which corresponds to the nominal vehicle speeds $\bar{u}_{1}>0$ and $\bar{u}_{2}<0$. The dynamics system has been linearised around the fixed point $\left(\bar{\omega}, \bar{u}_{1}\right)$, leading to the plant transfer function in $s$-domain with the following form:

$$
G_{p}(s)=\frac{k}{s+p}
$$

where $k=2 \bar{X}_{\omega^{2}} \bar{\omega}-\bar{X}_{u \omega} \bar{u}_{1}>0$ and $p=2 \bar{X}_{u^{2}} \bar{u}_{1}+\bar{X}_{u \omega} \bar{\omega}>$ 0 . Now that a simple expression of the dynamic model has been inferred, a simple feedback controller can be designed. A proportional-integral (PI) controller can be set in order to impose some time-domain specifications during the dynamic response:

$$
G_{c}(s)=K_{p}\left(1+\frac{1}{T_{i} s}\right)
$$

In particular, the PI controller adds to the open-loop transfer function a pole in the origin of the s-plane and a zero in $-1 / T_{i}$. The dynamic response of the system is affected by the zero, which increases the overshoot. In order to reduce the influence of the zero, it is placed far from the origin in

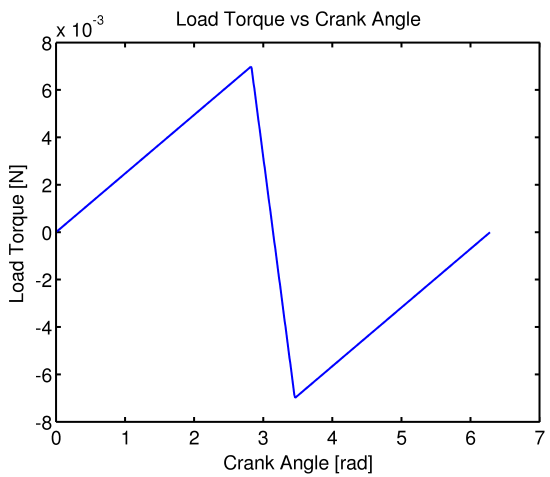

Fig. 5. In a single pulsation the load torque increases between 0 and $\pi$ radiant. At $\pi$ rad suddenly the cables start getting released and the load torque becomes negative.Between $\pi$ and $2 \pi$ radiant the load torque varies linearly reaching zero.

the left-half plane. In particular the zero has been chosen $n$ times greater than the pole $p$, i.e. $T_{i}=1 / n p$. The proportional gain $K_{p}$ is chosen by using the root locus technique in order to have an overshoot lower than $1 \%$, which corresponds to a settling time below 0.8 s to stay within $3 \%$ of the final value.

\section{Simulation Testing}

A preliminary test is performed in simulation in order to evaluate the controller (12) performance. The simulation is run considering the dynamic of the soft bodied underwater vehicle governed by equation (10) and the dynamic of a speed controlled motor. In particular, the motor controller implements an outer loop speed-controller and an inner loop current-controller. Both the speed and the current controllers use an anti-windup method to take into account the current and voltage limits of a DC-motor. The motor controllers have been designed applying the internal model principle in order to reject a disturbing load torque caused by the cables acting on the motor shaft. The load torque profile is a sum of ramp signals. The profile of load torque during a single pulsation (Fig. 5) is defined based on the following assumption. Between 0 and $\pi$ radiant the load torque increases linearly reaching the maximum value $C_{\max }$, as depicted in Fig. 4.a. At $\pi$ radiant suddenly the cables start getting released and the load torque becomes negative and equal to $-C_{\max }$, due to the pulling on the crank by the inflation of the silicone shell. Between $\pi$ and $2 \pi$ radiant the load torque varies between $-C_{\max }$ and 0 .

Considering the contraction experiments conducted in the air (Fig. 4.a) and the design specification according to which the maximum shortening of the cables with a $l=7 \mathrm{~mm}$ crank is $c=14 \mathrm{~mm}$, it occurs that the corresponding force for such contraction is $F_{c}=5 \mathrm{~N}$. This enables us to specify a maximum load torque $C_{\max }$ on the motor shaft equal to $C_{\max }=\lambda l F_{c}$, where $\lambda=2$ defines an arbitrary coefficient approximating the resistive contribution of water during the collapse of the silicone shell.

A clarification is needed concerning the phase of refill driven by the inflation of the shell. In the model presented here, it is assumed that the external wall of the silicone shell is inflating at the same rate at which the crank is releasing 


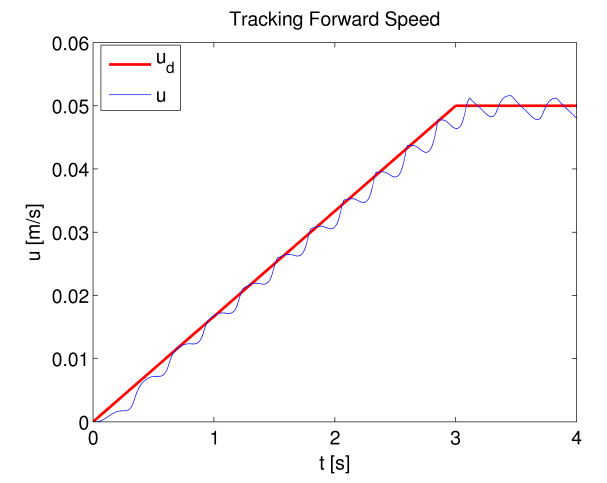

Fig. 6. Forward speed tracking of a soft bodied underwater vehicle: $u_{d}$ is the desired speed and $u$ is the real speed.

the cables. This guarantees that the cable slack problem is neglected and that the shell is always in its unstrained state at the time when the crank crosses the $\alpha=0$ spot. In practice it is found that the inflation of the shell requires a certain time to occur, dependent on the geometrical and material characteristics of the shell. Hence, if the crank is revolving at high angular speed, it may happen that the crank crosses the $\alpha=0$ point before the shell has fully inflated. This causes the motor to run idle until the cables are tensed again. This behaviour, which is disregarded in the present state of this work, plays a significant effect on the production of thrust since the time the shell requires to refill affects the time during which thrust cannot be generated and such effect will request special acknowledgement in the future development of the control scheme.

\section{RESUlts}

The performance of the robot and motor controllers are evaluated considering error signals, control signals and disturbance rejection. The performance evaluation follows the control chain from the robot controller to the inner loop current-controller.

The reference signal of the robot control system is a trapezoidal speed profile $u_{d}$. The Fig. 6 shows the speed tracking of the desired profile. The small oscillations around the desired signal depend on the jet-pulsed nature of the thruster. Indeed, the error between the desired and the real speed stays within $4 \%$ of the desired value.

The control signal of the robot controller provides the desired speed to the motor controllers. In particular, a commercial DC-motor is considered with a nominal voltage $v_{n}=6 \mathrm{~V}$, a nominal current $i_{n}=0.72 \mathrm{~A}$, a reduction gear $N_{r}=54$, and a reduced nominal speed $\omega_{n}=26 \mathrm{rad} / \mathrm{s}$.

The motor controllers have been designed to follow the desired speed $\omega_{d}$ and to compensate the load torque exerted by the cables. An optimal tracking of the motor speed and a perfect rejection of the load torque disturbance is realised by the motor controllers (Fig. 7). The control signals of the motor speed and the current controller are respectively the current and the voltage. During the simulation, the voltage is

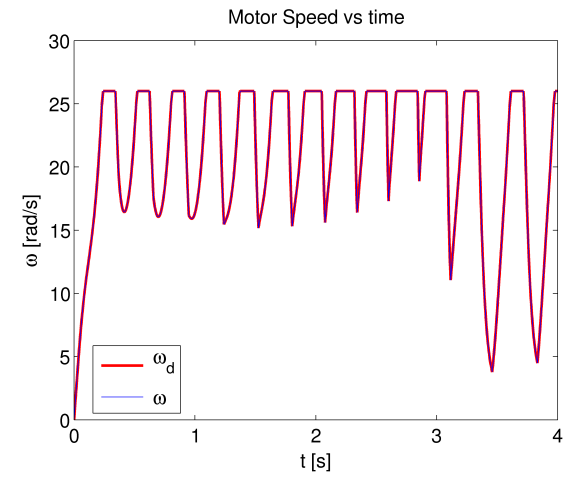

Fig. 7. Motor speed tracking: $\omega_{d}$ is the desired motor speed and $\omega$ is the real motor speed. The motor controllers compensate perfectly the load torque disturbances.

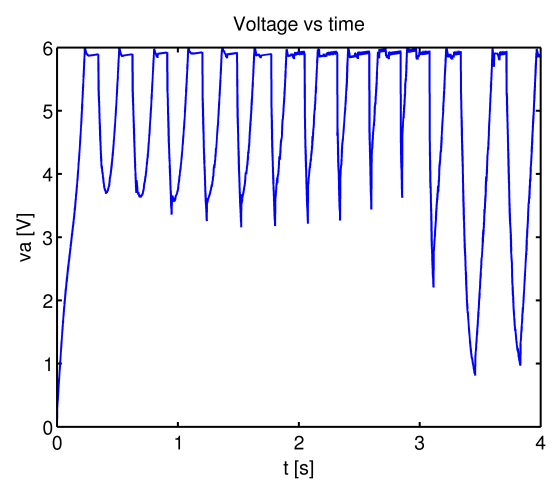

Fig. 8. The voltage applied to the motor by the inner-loop current controller.

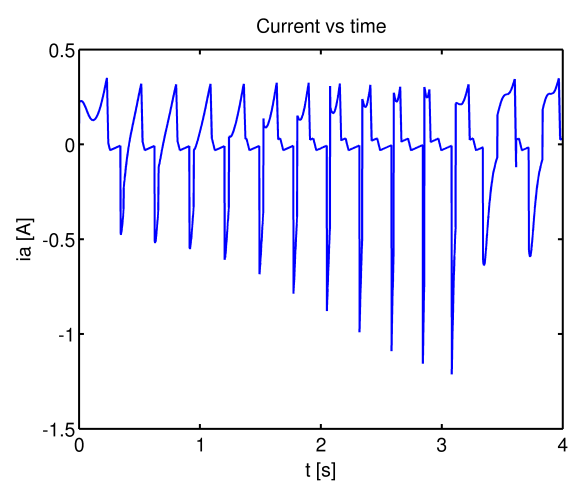

Fig. 9. the current drained by the motor. During a pulsation the current becomes negative because a negative load torque is applied to the motor shaft.

maintained always positive and bounded by the $v_{n}$ saturation limit (Fig. 8). It is mean the motor rotate always in the same direction. Whereas, during the jet pulsations the current signal becomes negative because a negative load torque acts on the motor shaft (Fig. 9). In this case, a saturation current limit equals to $2 i_{n}$ is allowed by the outer-loop speed controller.

Finally, the speed controlled motor generated the jet-pulsed thrust, as shown in Fig. 10. The amplitude of thrust pulsations increases during the acceleration phase of the robot, and it decreases reaching a stable value when the speed is constant. 


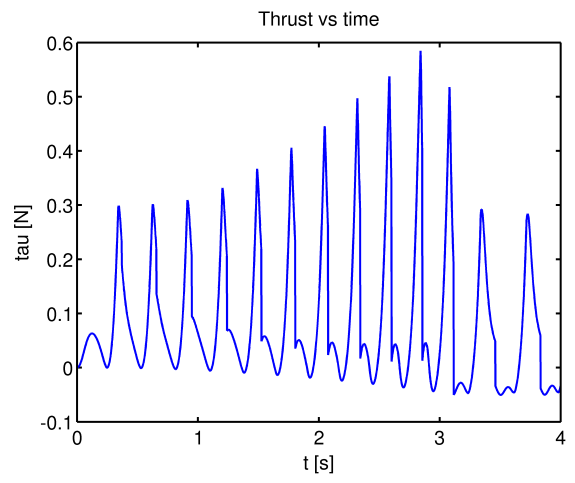

Fig. 10. The pulsed thrust generated by the speed controlled motor when the trapezoidal speed profile is fed into the robot control system.

\section{CONCLUSION}

A forward speed control for a pulsed-jet soft-bodied underwater vehicle is developed and tested for the first time. These preliminary results prove the effectiveness of the controller based on the soft robot linearised dynamics. Furthermore, the achieved results open a way for a set of further improvements such as a minimization of regime response oscillations implementing non-linear control technique, a reduction of the latency time during refill of the shell avoiding the slack problem, a compensation of shape change effects on the added mass and kinetic energy recovery due to added mass variation by using robust control techniques.

\section{REFERENCES}

[1] P.R. Bandyopadhyay, "Trends in Biorobotic Autonomous Undersea Vehicles", IEEE Journal of Oceanic Engineering, vol. 30, 2005.

[2] J. Conte, Y. Modarres-Sadeghi, M.N. Watts., F.S. Hover and M.S Triantafyllou, "A Fast-Starting Robotic Fish that Accelerates at $40 \mathrm{~ms}^{-2}$ ", Journal of Bioinspiration and Biomimetics, vol. 5, 2010.

[3] G.D. Weymouth and M.S. Triantafyllou, "Ultra-fast escape of a deformable jet-propelled body", Journal of Fluid Mechanics, vol. 721, 367385, 2013.

[4] M. Krieg and K. Mohseni, "Thrust characterization of a bio-inspired vortex ring generator for locomotion of underwater robots", IEEE Journal of Ocean Engineering, vol. 33, 542-554, 2008.

[5] L. A. Ruiz and R. W. Whittlesey and J. O. Dabiri, "Vortex-enhanced propulsion", Journal of Fluid Mechanics, vol. 668, 5-32, 2011.

[6] A. A. Moslemi and P. S. Krueger, "Propulsive efficiency of a biomorphic pulsed-jet underwater vehicle", Journal of Bioinspiration and Biomimetics, vol. 5, 1-14, 2010.

[7] F. Giorgio Serchi, A. Arienti and C. Laschi, "Biomimetic Vortex Propulsion: Toward the New Paradigm of Soft Unmanned Underwater Vehicles", IEEE/ASME Transactions on Mechatronics, vol. 18, 484-493, 3013.

[8] F. Giorgio Serchi, A. Arienti, I. Baldoli and C. Laschi, "An elastic pulsedjet thruster for Soft Unmanned Underwater Vehicles", IEEE International Conference on Robotics and Automation, May 6-10, 2013.

[9] P. S. Krueger and M. Gharib, "The significance of vortex ring formation to the impulse and thrust of starting jet", Physics of Fluid, vol. 15, 12711281, 2003.

[10] M. Krieg and K. Mohseni, "Dynamic modeling and control of biologically inspired vortex ring thrusters for underwater robot locomotion", IEEE Transactions on robotics, vol. 26, 542-554, 2010. 